Proceedings of the 42th "Jaszowiec" International School and Conference on the Physics of Semiconductors, Wisła 2013

\title{
Influence of Dephasing and Geometrical Parameters on Quantum Correction to DC Conductance of Cylindrical Nanowires
}

\author{
B.J. SPISAK*, M. Woloszyn And P. WóJCIK \\ AGH University of Science and Technology, Faculty of Physics and Applied Computer Science \\ al. A. Mickiewicza 30, 30-059 Kraków, Poland
}

\begin{abstract}
Calculations of the quantum correction to the DC conductance of a cylindrical nanowire due to the quantum interference are presented. The real space Cooperon equation is solved for cylindrical geometry. Using this approach, it is shown that the quantum correction to the conductance in the weak localisation regime depends not only on the dephasing processes but also on geometrical parameters of the nanowire.
\end{abstract}

DOI: 10.12693 /APhysPolA.124.838

PACS: 72.15.Rn, 72.80.Ey, 73.63.Nm

\section{Introduction}

The progress made in the development of the experimental techniques of low-dimensional system fabrication allows to synthesize semiconductor nanowires with increasing aspect ratio of a rod (length:diameter). Nowadays, the length of semiconductor nanowires exceeds tens of micrometers and their diameter is below $100 \mathrm{~nm}[1-3]$. The transport properties of nanowires based on III-V and II-VI compound semiconductor materials can be controlled by adding impurities during the growth process of nanostructures $[2,3]$. Simultaneously, these impurities form scattering centres for carriers, and their multiple scatterings lead to momentum correlations, so that the electronic transport displays the non-Markovian properties, i.e., successive scatterings of carriers are not independent. One of the main consequences of these correlations in weakly disordered systems is the phase coherence of carriers, which leads to the weak localisation phenomena $[4,5]$.

A key parameter for the investigation of the coherence property of electronic transport is a dephasing length that describes the distance after which the phase coherence of carriers is loosened by their coupling with phonons, magnetic impurities, other carriers or external electromagnetic fields.

In this report we discuss the effect of dephasing on the electronic transport in the cylindrical semiconductor nanowires within the limit of weak disorder. We develop the theory of weak localization for cylindrical geometry, and we apply it to calculate the DC conductance of the nanowire as a function of the reduced effective length, which is defined as the ratio of the nanowire length to the dephasing length, for different values of the aspect ratio.

\footnotetext{
*corresponding author; e-mail: bjs@agh.edu.pl
}

\section{Model, assumptions and method of calculations}

Let us consider spinless electron gas confined to a three-dimensional nanowire made of a semiconductor material with randomly distributed point-like scattering centres. We assume that the potential which confines the conduction electrons in nanowire possesses the rotational symmetry along growth axis, so that the cylindrical shape of nanowire is considered. Its geometrical parameters are: radius $\rho_{\mathrm{c}}$ and length $L_{z}$. The transport properties of the nanosystem can be characterised by the electrical conductivity which depends not only on semiconductor materials but also on the strength of disorder due to the randomly distributed scattering centres. In the present case, we assume that weak disorder is described by the Gaussian model, and the scattering centres correlator possesses the white-noise property. The electronic transport in the weakly disordered nanosystems is modified by the quantum interference which is responsible for the electronic coherence. The coherent part enhances the classical return probability and leads to the quantum correction to the conductance in the form

$$
\Delta G(\omega)=-\frac{2 e^{2} D_{0} \tau}{\pi \hbar} \frac{1}{L_{z}^{2}} \int \mathrm{d}^{3} r \mathcal{C}(\boldsymbol{r}, \boldsymbol{r} ; \omega),
$$

where $D_{0}=v_{\mathrm{F}}^{2} \tau / 3$ is the diffusion coefficient, $v_{\mathrm{F}}$ is the Fermi velocity, $\tau$ is the elastic relaxation time, and the Cooperon $\mathcal{C}(\boldsymbol{r} ; \boldsymbol{r})$ represents short-range contribution to the return probability. The real space Cooperon equation in the presence of phase-breaking processes is [6]:

$$
\begin{aligned}
& {\left[\frac{\partial}{\partial t}-D_{0} \nabla_{\boldsymbol{r}}^{2}+\frac{1}{\tau_{\phi}}\right] \mathcal{C}\left(\boldsymbol{r}, t ; \boldsymbol{r}^{\prime}, t^{\prime}\right)} \\
& \quad=\frac{1}{\tau} \delta\left(\boldsymbol{r}-\boldsymbol{r}^{\prime}\right) \delta\left(t-t^{\prime}\right),
\end{aligned}
$$

where $\tau_{\phi}$ is the dephasing time. The boundary conditions for Eq. (2) may be written as

$$
\left.\mathcal{C}\left(\boldsymbol{r}, t ; \boldsymbol{r}^{\prime}, t^{\prime}\right)\right|_{\text {boundary }}=0,
$$




$$
\left.\frac{\partial}{\partial r} \mathcal{C}\left(\boldsymbol{r}, t ; \boldsymbol{r}^{\prime} t^{\prime}\right)\right|_{\text {boundary }}=0 .
$$

The form of the boundary conditions corresponds to the absence of diffusion of conduction electrons through the lateral surface of the cylindrical nanowire, however current flows along the axis of nanowire which is embedded between reflectionless electron reservoirs. The Fourier transform with respect to $t-t^{\prime}$ applied to Eq. (2) allows us to convert it to the form

$$
\left[-\mathrm{i} \omega \tau-D_{0} \tau \nabla_{\boldsymbol{r}}^{2}+\tau \tau_{\phi}^{-1}\right] \mathcal{C}\left(\boldsymbol{r}, \boldsymbol{r}^{\prime} ; \omega\right)=\delta\left(\boldsymbol{r}-\boldsymbol{r}^{\prime}\right),
$$

where $\omega$ is a frequency. The general solution of Eq. (4) can be written in the spectral form as follows:

$$
\mathcal{C}\left(\boldsymbol{r}, \boldsymbol{r}^{\prime} ; \omega\right)=\sum_{j} \frac{\mathcal{Q}_{j}^{*}\left(\boldsymbol{r}^{\prime}\right) \mathcal{Q}_{j}(\boldsymbol{r})}{-\mathrm{i} \omega \tau+\tau \tau_{\phi}^{-1}+\lambda_{j}},
$$

where $\mathcal{Q}_{j}(\boldsymbol{r})$ and $\lambda_{j}$ are eigenfunctions and eigenvalues, respectively, which satisfy the equation

$$
\left[-\mathrm{i} \omega \tau-D_{0} \tau \nabla_{\boldsymbol{r}}^{2}+\tau \tau_{\phi}^{-1}\right] \mathcal{Q}_{j}(\boldsymbol{r})=\lambda_{j} \mathcal{Q}_{j}(\boldsymbol{r}) .
$$

In the considered case, the strategy of determination of the quantum correction to the conductance (1) is based on the solution of eigenvalue problem (6) with boundary conditions (3). It allows us to find the spectral form of Cooperon (5), and then compute the quantum correction to the DC conductance (1) for $\boldsymbol{r}^{\prime}=\boldsymbol{r}$. For this purpose, we solve the eigenvalue Eq. (6) in the cylindrical coordinates and in the limit of $\omega \rightarrow 0$, using the separation of variables method, i.e., $\mathcal{Q}(\boldsymbol{r})=\mathcal{R}(r) \mathcal{F}(\varphi) \mathcal{Z}(z)$ is assumed. The eigenfunctions $\mathcal{Q}_{q n m}(r, \varphi, z)$ corresponding to the eigenvalues $\lambda_{q n m}$ can be written as follows:

$$
\mathcal{Q}_{q n m}(r, \varphi, z)=A_{q n m} \mathcal{R}_{m}\left(\gamma_{q m} r\right) \mathcal{F}_{m}(\varphi) \mathcal{Z}_{n}\left(k_{n} z\right),
$$

where $\mathcal{Z}_{k_{n}}(z) \sim \sin k_{n} z$ with $k_{n}=n \pi / L_{z}$ and $(n=$ $1,2, \ldots), \mathcal{F}_{m}(\varphi) \sim \exp (\mathrm{i} m \varphi)$ with $(m=0, \pm 1, \ldots)$, $\mathcal{R}_{m}(\gamma r) \sim J_{m}(\gamma r)$, and $A_{q n m}$ represents a normalisation constant. The quantity $\gamma$ is determined by the solution of transcendental equation in the form

$$
\frac{m}{\rho_{\mathrm{c}}} J_{m}\left(\gamma \rho_{\mathrm{c}}\right)-\gamma J_{m+1}\left(\gamma \rho_{\mathrm{c}}\right)=0,
$$

where symbol $J_{l}(\gamma r)$ denotes the Bessel function of the first kind of integral order $l(l=m$ or $l=m+1)$. Solutions of the transcendental Eq. (8) are given by the enumerable set of discrete numbers $\eta_{q m}\left(\rho_{\mathrm{c}}\right)$ with index $q$ numbering the solutions. The above procedure leads to the set of eigenvalues in the form

$$
\lambda_{q n m}=D_{0} \tau\left\{\left[\frac{\eta_{q m}\left(\rho_{\mathrm{c}}\right)}{\rho_{\mathrm{c}}}\right]^{2}+\left(\frac{n \pi}{L_{z}}\right)^{2}\right\} .
$$

\section{Results and discussion}

Investigation of the quantum corrections to conductance due to quantum interference effects in the weakly disordered nanowires requires to satisfy a condition in the form

$$
\lambda_{\mathrm{F}} \ll \ell \ll L_{z} \ll L_{\phi},
$$

where $\lambda_{\mathrm{F}}$ is the Fermi wavelength, $\ell$ is the mean free path, and $L_{\phi}=\sqrt{D_{0} \tau_{\phi}}$ is the dephasing length. Accord- ing to the results presented in the previous section, the relative correction to the $\mathrm{DC}$ conductance of the cylindrical nanowire in this regime of electronic transport is given by the formula

$$
\frac{\Delta G}{G_{0}}=-\sum_{q, n, m} \frac{2}{\left(\frac{L_{z}}{L_{\phi}}\right)^{2}+\left[\frac{L_{z}}{\rho_{\mathrm{c}}} \eta_{q m}\left(\rho_{\mathrm{c}}\right)\right]^{2}+(n \pi)^{2}},
$$

where $G_{0}=2 e^{2} / h$ is the quantized unit of electrical conductance, $L_{z} / \rho_{\mathrm{c}}=\alpha$ is the aspect ratio of the nanowire, and $L_{z} / L_{\phi}$ represents the reduced effective length. In the context of the weak localisation contribution to the DC conductance, the semiconductor nanowires made of In and As elements are particularly interesting because the ballistic as well as drift transport regime is observed in these nanostructures, depending on the geometrical parameters [7].

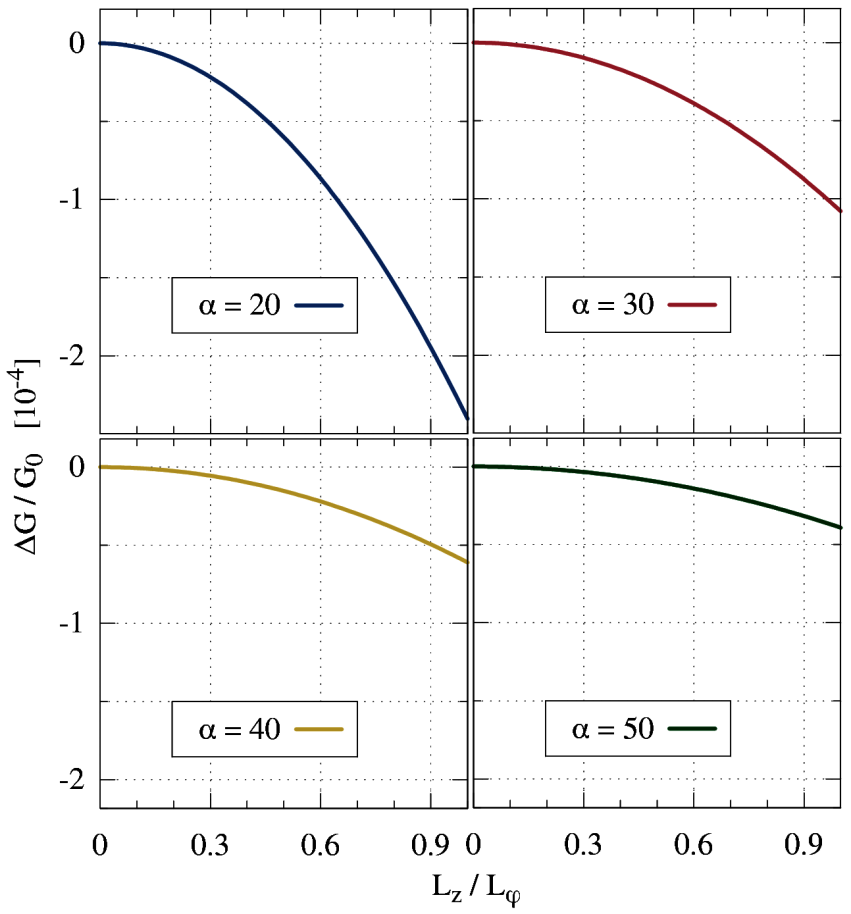

Fig. 1. The relative correction to DC conductance of the cylindrical nanowire as a function of the reduced effective length $L_{z} / L_{\phi}$.

Figure 1 displays the results of calculations of the relative corrections to the DC conductance as a function of the reduced effective length for different values of the aspect ratio. In these calculations, we assume, according to Refs. $[7,8]$, that the radius of InAs nanowire is $80 \mathrm{~nm}$, and the mean free path $\ell=40 \mathrm{~nm}$. In all cases the quantum corrections are decreasing functions of the reduced effective length independently of the aspect ratio. The calculated weak localisation corrections are of order $10^{-4}$ and cause a decrease of the DC conductance of the cylindrical nanowire because the sign of this correction is negative. 


\section{Conclusions}

We derived the form of the quantum correction due to quantum interference effects to the DC conductance of weakly disordered cylindrical nanowires in the weak localisation regime. For this purpose, we solved the real space Cooperon equation with the phase breaking ratio in the cylindrical geometry. The main result of our calculations is the formula (11). We showed that this formula can be simply expressed by aspect ratio of the nanowire and reduced effective length. The derived quantum corrections reduce the $\mathrm{DC}$ conductance of the nanowire and are sensitive to the dephasing processes and geometrical parameters of the cylindrical nanowire.

\section{Acknowledgments}

This project is supported by the National Science Centre, Poland under grant DEC-2011/03/B/ST3/ 00240 .

\section{References}

[1] F.M. Ross, Rep. Prog. Phys. 73, 114501 (2010).

[2] R. Rurali, Rev. Mod. Phys. 82, 427 (2010).

[3] H.J. Joyce, Q. Gao, H.H. Tan, C. Jagadish, Y. Kim, J. Zou, L.M. Smith, H.E. Jackson, H.E.J.M. Yarrison-Rice, P. Parkinson, M.B. Johnston, Prog. Quant. Electron. 35, 23 (2011).

[4] L.P. Gor'kov, A.I. Larkin, D.E. Khmel'nitskii, JETP Lett. 30, 228 (1979).

[5] P.A. Lee, T.V. Ramakrishnan, Rev. Mod. Phys. 57, 287 (1985).

[6] J. Rammer, Quantum Field Theory of Nonequilibrium States, Cambridge University Press, Cambridge 2007.

[7] X. Zhou, S.A. Dayeh, D. Aplin, D. Wang, E.T. Yu, Appl. Phys. Lett. 89, 053113 (2006).

[8] M. Scheffler, S. Nadj-Perge, L.P. Kouwenhoven, M.T. Borgström, E.P.A.M. Bakkers, J. Appl. Phys. 106, 124303 (2009). 